\title{
Revascularização do miocárdio no idoso: fatores de risco para morbidade e mortalidade hospitalar
}

\author{
José Carlos R. IGLÉZIAS*, Luís Alberto DALLAN*, Sérgio Ferreira de OLIVEIRA*, Antônio F. RAMIRES*,
} Sérgio Almeida de OLIVEIRA*, Geraldo VERGINELLI*, Adib D. JATENE*.

IGLÉZIAS, J. C. R.; DALLAN, L. A.; OLIVEIRA, S. F.; RAMIRES, A. F.; OLIVEIRA S. A.; VERGINELLI, G.; JATENE, A. D. - Revascularização do miocárdio no idoso: fatores de risco para morbidade e mortalidade hospitalar. Rev. Bras. Cir. Cardiovasc., 6(3):203-207, 1991.

RESUMO: Com a finalidade de determinar os principais fatores de risco para a morbidade e mortalidade hospitalar de pacientes coronarianos idosos (idade > 65 anos), submetidos a revascularização isolada, eletiva do miocárdio, estudamos prospectivamente 72 pacientes consecutivos, que possuíam observaçōes completas no InCór, no período compreendido entre janeiro e dezembro de 1988 . No roteiro do protocolo foram incluídos fatores clínicos, radiológicos, hemodinâmicos, operatórios e de pós-operatórios (Tabela 1). Os resultados foram analisados utilizando-se o teste do Qui quadrado de Pearson e a Prova Exata de Fisher, através do SPSS (Statistical Package for Social Science). Dentre os fatores analisados, apresentaram significância estatística: o tabagismo, o número de vasos coronários acometidos, o tempo de duraçäo da circulaçäo extracorpórea, o tempo de pinçamento da aorta, o número total de enxertos realizados, a presença de anastomose mamária coronária e o índice de movimentação da parede ventricular esquerda.

DESCRITORES: miocárdio, revascularizaçāo, pacientes idosos; miocárdio, revascularizaçāo, fatores de risco.

\section{INTRODUÇĀO}

A avaliação objetiva do tratamento da doença arterial coronária é baseada no completo conhecimento dos efeitos clínicos e na história natural da moléstia. O diagnóstico da doença arterial coronária tem por base a observação das alteraçōes anatômicas da circulação arterial do coração do paciente. A expressão clínica da mudança anatômica das coronárias, a isquemia ou a doença isquêmica do coração despertam a atenção do médico. Nos pacientes com diagnóstico clínico de doença arterial coronária, sabe-se classicamente que a angina do peito e o infarto do miocárdio são as manifestaçōes clínicas mais importantes; a angina aparce em $38 \%$ das mulheres, enquanto o infarto do miocárdio é a primeira manifestação nos homens $(42 \%)$, aparecendo somente em $21 \%$ das mulheres.
A mortalidade anual decorrente da angina do peito em pacientes tratados clinicamente, permaneceu consideravelmente estável até a década de 60 , de acordo com diversos estudos.

Em 1956, RICHARDS et alii ${ }^{12}$ relataram mortalidade anual de $4 \%$ em pacientes seguidos durante 25 anos. KANNEL \& FEINLEIB ${ }^{8}$ relataram taxas de mortalidade idênticas. FRANK et alii4 indicaram que os pacientes anginosos podem ser separados em grupos de alto e baixo risco. Assim, quando a pressão arterial e o eletrocardiograma eram normais, 0 risco de mortalidade era de $8 \%$ ao ano, risco considerado alto quando comparado com $1,8 \%$ de mortalidade a cada ano quando essas variáveis clínicas eram normais.

Tanto os vasodilatadores de ação curta, como

Trabalho realizado no Instituto do Coração do Hospital das Clínicas da Faculdade de Medicina da Universidade de São Paulo. São Paulo, SP, Brasil. Recebido para publicação em $1^{\circ}$ de dezembro de 1991.

* Do Instituto do Coração do Hospital das Clínicas da Faculdade de Medicina da Universidade de São Paulo.

Endereço para separatas: José Carlos Iglézias. Av. Dr. Eneas de Carvalho Aguiar, 44. Divisão Cirúrgica. 05403 São Paulo, SP, Brasil. 
IGLÉZIAS, J. C. R.; DALLAN, L. A.; OLIVEIRA, S. F.; RAMIRES, A. F.; OLIVEIRA S. A.; VERGINELLI, G.; JATENE, A. D. Revascularizaçāo do miocárdio no idoso: fatores de risco para morbidade e mortalidade hospitalar. Rev. Bras. Cir. Cardiovasc., 6(3):203-207, 1991.

TABELA 1

FATORES INCLUIDOS NO ROTEIRO DO PROTOCOLO

\section{Idade}

2 Sexo

3 Moléstias associadas à doença coronária

- diabétes melito

- acidente vascular cerebral prévio

- doença pulmonar obstrutiva

- hipertensäo arterial sistêmica

- tabagismo

- obesidade índice de massa corpórea $\geqslant 30$

- doença arterial vascular periférica

- dislipidemia

4 Angina instável

5 Classe funcional para insuficiência cardíaca congestiva (NYHA) pré-operatória

6 Infarto do miocárdio pré-operatório

7 Índice cardiotorácico

8 Pressão diastólica final do ventrículo esquerdo (préoperatório)

9 Acometimento do tronco de coronária esquerda

10 Número de enxertos realizados

11 Revascularização com artéria torácica interna (mamária interna)

12 Tipo de proteção miocárdica

13 Tempo de duração da circulação extracorpórea

14 Tempo de pinçamento aórtico

15 Presença de baixo débito no pós-operatório

16 Uso de baläo intra-aórtico

17 Acidente vasculas cerebral no pós-operatório

18 Induficiência renal no pós-operatório (creatinina $3,0 \mathrm{mg} / \mathrm{dl}$ )

19 Infecção no pós-operatório

20 Assistência ventilatória por período 24 horas

21 Permanência hospitalar em dias

22 Situaçāo após 30 dias

os de ação prolongada, usados para o alívio da angina, provaram ser efetivos também no alívio da isquemia miocárdica.

O risco de eventos cardíacos após o infarto agudo do miocárdio fica significativamente reduzido, após a interrupção do hábito de fumar ${ }^{3,13}$.

Os agentes antiadesivos plaquetários também parecem alterar favoravelmente o prognóstico da doença arterial coronária; entretanto, estudos cooperativos de diversos autores, recentes e completos, falharam em demonstrar o efeito benéfico da aspirina no prognóstico da doença coronária aterosclerótica. A administração de agentes bloqueadores beta-adrenérgicos tem reduzido a mortalidade dos pacientes acometidos por infarto agudo do miocárdio.

Outros tratamentos que podem ser benéficos aos pacientes portadores de doença arterial coronária incluem manipulação de dieta, redução do nível sangüineo de determinados lípides e exercícios físicos regulares e vigorosos.

O declínio da mortalidade nas doenças cardiovasculares e as mudanças recentes introduzidas no tratamento clínico da angina do peito devem ser considerados na avaliaçāo do tratamento cirúrgico da doença arterial coronária.

Fatores adicionais às manifestaçōes clínicas que se correlacionam com o prognóstico da doença arterial coronária incluem idade do paciente, tamanho do coração, função dos ventrículos, tolerância ao exercício físico e a extensão das lesões coronárias ${ }^{7,8}$.

Baseado nos dados da literatura e com o objetivo de identificar os fatores de risco para a nossa população, elaboramos um estudo prospectivo levando em consideração fatores clínicos, radiológicos, hemodinâmicos, operatórios e de pós-operatório (Tabela 2).

TABELA 2

VARIÁVEIS ANALISADAS COM $P<0,05$

\section{Tabagismo}

Ramos arteriais acometidos por aterosclerose

Índice de movimentação da parede ventricular esquerda

- Tempo de duração da circulação extracorpórea

- Tempo de duraçāo do pinçamento aórtico

- Número total de enxertos realizados

- Presença de anastomose mamária-coronária

\section{CASUÍSTICA E MÉTODOS}

Com a finalidade de cumprir nossa proposição estudamos, prospectivamente, no Instituto do Coração do Hospital das Clínicas da Faculdade de Medicina da Universidade de São Paulo, no período compreendido entre janeiro e dezembro de 1988, 72 pacientes portadores de doença arterial coronária obstrutiva crônica, com idade > 65 anos, que possuíam observaçöes completas e foram submetidos a revascularização eletiva isolada do miocárdio.

Os resultados obtidos foram analisados utilizando-se o teste do Qui quadrado de Pearson, a Prova Exata de Fisher e o programa SPSS (Statistical Package for Social Science).

\section{RESULTADOS}

Dos 72 pacientes estudados, $59(81,94 \%)$ eram do sexo masculino e a média de idade foi igual a 68,84 anos (65 a 75). O diabetes melito foi constatado em 28 (38,88\%). Em $13(18,05 \%)$ pacientes 
IGLÉZIAS, J. C. R.; DALLAN, L. A.; OLIVEIRA, S. F.; RAMIRES, A. F.; OLIVEIRA S. A.; VERGINELLI, G.; JATENE, A. D. Revascularizaçäo do miocárdio no idoso: fatores de risco para morbidade e mortalidade hospitalar. Rev. Bras. Cir. Cardiovasc., 6(3):203-207, 1991.

foi constatada a doença vascular cerebral, sendo que em cinco $(6,94 \%)$ havia referência a acidente vascular cerebral prévio. A doença vascular periférica foi constatada em $11(15,27 \%)$ pacientes.

A doença pulmonar obstrutiva crônica foi constatada em $16(22,22 \%)$ pacientes. A hipertensão arterial sistêmica foi constatada em 36 (50\%) pacientes.

O índice de massa corpórea ficou entre 30 e 40 em oito $(11,11 \%)$ pacientes. Quanto às moléstias associadas à coronariopatia, $17(23,61 \%)$ pacientes apresentavam uma, $24(33,33 \%)$ apresentavam duas, $11(15,27 \%)$ apresentavam três, $13(18,05 \%)$ apresentavam quatro, em um $(1,28 \%)$ cinco moléstias associadas à doença de base.

Em relação ao tabagismo, $28(38,88 \%)$ pacientes nunca haviam feito uso de cigarros. A angina do peito esteve presente em $33(45,83 \%)$ pacientes do grupo.

Quanto à classe funcional (c. f.), para angina do peito, $16(22,22 \%)$ se encontravam em c.f.I, 49 $(68,05 \%)$ em c.f. II e sete $(9,72 \%)$ em c. f. III.

O infarto do miocárdio foi constatado no eletrocardiograma pré-operatório de repouso em 40 $(55,55 \%)$ pacientes.

Os estertores crepitantes foram constatados no exame físico de entrada em sete $(9,72 \%)$ pacientes.

O índice cardiotorácico foi $\leqslant 0,50$ em $46(63,88 \%)$ pacientes da série.

Os niveis de creatinina foram $\geqslant 3,0 \mathrm{mg} / \mathrm{dl}$ em dois $(2,72 \%)$ pacientes no pré-operatório.

Em relação aos vasos coronários acometidos, cinco $(6,94 \%)$ pacientes eram uniarteriais, 20 $(27,77 \%)$ eram biarteriais e $47(65,27 \%)$ eram triarteriais. A média de vasos acometidos por pacientes foi igual a 2,58 .

A pressāo diastólica final do ventrículo esquerdo foi $\geqslant 20 \mathrm{~mm}$ de $\mathrm{Hg}$ em 24 (33,33\%) pacientes da série.

A fração de ejeção calculada através do ecocardiograma bidimensional foi $\geqslant 60 \%$ em 51 (70,83\%) pacientes.

O tronco da coronária esquerda apresentava estenose maior que $30 \%$ em sete $(9,72 \%)$ pacientes, sendo que em quatro $(5,55 \%)$ deles o comprometimento era $>70 \%$.

Em relação ao índice de movimentação da parede ventricular esquerda, podemos dizer que foi > 10 em $19(26,38 \%)$ pacientes do grupo.

A circulação extracorpórea durou menos que 120 minutos em 54 (75\%) pacientes e a média de tempo foi igual a $105,52(30-195)$ minutos.

O tempo de pinçamento aórtico foi menor que 90 minutos em $62(86,11 \%)$ pacientes, sendo que a média foi igual a 63,20 (14-140) minutos.

A proteção miocárdica foi realizada por meio de solução cardioplégica em $29(40,27 \%)$ pacientes e nos $43(59,72 \%)$ restantes, através do pinçamento aórtico intermitente.

A média de enxertos por paciente foi igual a $2,75(1-5)$.

Trinta e oito $(52,77 \%)$ pacientes receberam uma anastomose mamária-coronária e a média por paciente foi de $0,58(0-2)$.

A permanência hospitalar foi igual a 25,16 (458) dias

Após 30 dias, seis $(8,33 \%)$ pacientes tinham ido a óbito e $66(91,66 \%)$ permaneciam vivos, sendo que, destes, nove $(12,5 \%)$ apresentavam seqüelas.

\section{COMENTÁRIOS}

O declínio da mortalidade nas doenças cardiovasculares e as mudanças recentes introduzidas no tratamento clínico da angina do peito devem ser considerados na avaliçāo do tratamento cirúrgico da doença arterial coronária. Fatores adicionais às manifestações clínicas que se correlacionam com $\checkmark$ prognóstico da doença, segundo BRUSCHKE et alii ${ }^{1}$ e KANNEL et alii ${ }^{\circ}$, incluem: idade do paciente, tamanho do coração, função dos ventrículos, tolerância ao exercício físico e extensão das lesões coronárias. Em nossa série, não houve correlação entre morbidade e mortalidade com o fator idade isoladamente. Assim, como os autores, encontramos correlação entre parâmetros relativos à disfução ventricular esquerda.

KENNEDY et alii ${ }^{10}$, analisando 6176 pacientes portadores de doença arterial coronária, selecionaram algumas variáveis individuais de risco que consideraram como tendo valor prognóstico para a mortalidade operatória. Em ordem decrescente de importância, foram: idade, estenose do tronco da coronária esquerda ( $>90 \%$ de sua luz), sexo feminino, movimentação da parede ventricular esquerda, pressão diastólica final do ventrículo esquerdo e ausculta de estertores crepitantes nas bases pulmonares. Com exceção do índice de movimentação da parede ventricular esquerda, os demais fatores foram confirmados em nossa série.

As taxas de mortalidade dos pacientes do sexo masculino, portadores de doença arterial coronária são muito mais altas do que as das mulheres, sendo que, nos primeiros, a taxa de mortalidade é 5,2 
IGLÉZIAS, J. C. R.; DALLAN, L. A.; OLIVEIRA, S. F.; RAMIRES, A. F.; OLIVEIRA S. A.; VERGINELLI, G.; JATENE, A. D. Revascularizaçāo do miocárdio no idoso: fatores de risco para morbidade e mortalidade hospitalar. Rev. Bras. Cir. Cardiovasc., 6(3):203-207, 1991.

vezes maior, considerando a faixa etária de 35 a 45 anos. Na faixa etária entre 65 e 74 anos, também ocorre maior mortalidade no sexo masculino, sendo que a taxa é 2,4 vezes maior que a encontrada para o sexo feminino. Em nossa análise, envolvendo pacientes com idade > 65 anos, não encontramos correlação significativa entre o sexo e a mortalidade hospitalar.

LOOP et alii ${ }^{11}$ e JONES et alii ${ }^{7}$ observaram que a estenose do tronco da coronária esquerda estava associada a uma mortalidade pós-operatória elevada.

Esses dados foram confirmados pelo estudo cooperativo multicêntrico da cirurgia das artérias coronárias ${ }^{2}$, porém o foi em nossa casuística.

GERSH et alii ${ }^{5,6}$, analisando a sobrevida tar- dia de 1086 pacientes com idade superior a 65 anos, observaram que o número de enxertos realizados nāo afetavam significativamente a sobrevida dos pacientes durante os cinco primeiros anos do pós-operatório. Em nossa casuística, encontramos correlação significativa em relação à mortalidade hospitalar, quando consideramos esses dois fatores.

Assim, no estudo realizado, constatamos significância estatística para a morbidade - mortalidade hospitalar nos seguintes ítens: tabagismo, número de vasos acometidos por aterosclerose, índice de movimentação da parede ventricular esquerda, tempo de duração da circulação extracorpórea, tempo de duração do pinçamente aórtico, número total de enxertos realizados e número de anastomoses mamária-coronária.

RBCCV 44205-152

IGLÉZIAS, J. C. R.; DALLAN, L. A.; OLIVEIRA, S. F.; RAMIRES, A. F.; OLIVEIRA S. A. O.; VERGINELLI, G.; JATENE, A. D. - Myocardial revascularization: risck factors of nosocomial morbidity and mortality in elderly. Rev. Bras. Cir. Cardiovasc., 6(3):203-207, 1991.

ABSTRACT: Seventy-two consecutive patients with complete observations were studied at the Heart Institute (InCór) between January and December 1988, to determine the main risk factors of nosocomial morbidity and mortality of elderly coronary patients ( $\geqslant 65$ years) submitted to elective isolated myocardial revascularization. Clinical, radiologic, hemodynamic, operative and post-operative factors were included in the protocol. The results were analysed through Pearson's $X^{2}$ test and Fisher's Exact Test using the SPSS (Statistical Package for Social Science). Among the analyzed factors, smoking habit, number of impaired coronary vessels, duration of extracorporeal circulation, time of aortic clamping, total number of performed grafts, presence of the coronary-mammary anastomisis and index of the left ventricular wall movement present statistical significance. factors.

DESCRIPTORS: myocardial revascularization, elderly patients; myocardial revascularization, risk

\section{REFERÊNCIAS BIBLIOGRÁFICAS}

BRUSCHKE, A. V. G.; PROUDFIT, W. L.; SONES, F. M. - I: Progress study of 590 consecutive non surgical cases of coronary artery disease followed 5-9 years. II: Ventrículo graphic and other correlations. Circulation, 47: 1154-1163, 1973.

CHAITMAN, B. R.; FISCHER, L. D.; BOURASSA, M. G.; DAVIS, K.; ROGERS, W. J.; MAYNARD, C.; TYRAS, D. H.; BERGER, R. L.; JUDKINS, M. P.; RINGOVIST, I.; MOCK, M. B.; KILLIP, T. - Effect of coronary bypass surgery on survival patterns in subsets of patients with left main coronary artery disease. Am. J. Cardiol., 48: 765-777, 1981. observations on male british doctors. 2: $1525-1536,1976$

Br. Med. J.,

4 FRANK, C. W.; WEIBLATT, E.; SHAPIRO, S. - Angina pectoris in men: prognostic significance of select medical factors. Circulation, 47: 509-517, 1973.

5. GERSH, B. J.; KRONNAL, R. A.; FRYE, R. L.; SCHAFF, H. V.; RYAN, T. J.; GOSSELIN, A. J.; KAISER, G.; KILLIP, T. - Coronary arteriography coronary artery bypass surgery morbility and mortality in patients ages 65 years or older. Circulation, 7: 483-491, 1983.

6 GERSH, B. J.; KRONNAL, R. A.; SCHAFF, H. V.; FRYE, R. L.; RYAN, T. J.; MYERS, W. O.; ATHEARN, M. W.; GOSSELIN, A. J.; KAISER, G. C.; KILLIP, T. - 
IGLÉZIAS, J. C. R.; DALLAN, L. A.; OLIVEIRA, S. F.; RAMIRES, A. F.; OLIVEIRA S. A.; VERGINELLI, G.; JATENE, A. D. Revascularizaçāo do miocárdio no idoso: fatores de risco para morbidade e mortalidade hospitalar. Rev. Bras. Cir. Cardiovasc., 6(3):203-207, 1991.

Long term (15 years) results of coronary bypass surgery in patients 65 years old or older: a report from the Coronary Artery Surgery Study. Circulation, 68 (Supl. 2): 190-199, 1983.

7 JONES, E. L.; CRAVES, J. M.; KING III, S. B.; DOUGLAS, J. S.; BRADFORD, J. M.; BROWN, C. M.; BONE, D. K.; HATCHER Jr., C. R. - Clinical, anatomical and functional descriptors influencing morbility survival and adequacy of revascularization following coronary bypass. Ann. Surg., 192:390-396, 1989.

8 KANNEL, W. B. \& FEINLEIB, M. - Natural history of angina pectoris in the Framingham study: prognosis and survival. Am. J. Cardiol., 29:154-163, 1962.

9 KANNEL, W. B. L.; McGeE. D.; GORDON, T. A. General cardiovascular risk profile: the Framingham study. Am. J. Cardiol., 38:46-51, 1976.

10 KENNEDY, J. W.; KAISER, G. C.; FISHER, L. D.; MAYNARD, C.; FRITS, J. K.; MYERS, W.; MUDD, J. G.; RYAN, T. J.; COGGING, J. - Multivariate discriminant analysis of the clinical and angiographic predictors of operative mortality from the Colaborative Study in Coronary Artery Surgery (CASS). J. Thorac. Cardiovasc. Surg.,80: 876-887, 1980.

11 LOOP, F. D.; LYTLE, B. W.; COSGROVE, D. M.; SHELDON, W. C.; IRARRAZAVAL, M.; TAYLOR, P. C.; GROVES, L. K.; RICHARD, A. D. - Atherosclerosis of the left main coronary artery: 5 years results of surgical treatment. Am. J. Cardiol., 44: 195-201, 1979.

12 RICHARDS, D. W.; BLAND, E. F.; WHITE, P. D. - A completed twenty five years follow-up study of 456 patients with angina pectoris. J. Chronic. Dis., 4: 423-433, 1956.

13 WILHELMSEN, C.; ELMFELDT, D.; VENDIN, J. A.; TRIBBLIN, G.; WILHELMSEN, L. - Smoking and miocardial infarction. Lancet, 1: 415-420, 1975.

14 WILHELMSEN, C.; WILHELMSEN, L.; VENDIN, J. A.; TRIBBLIN, G.; WERKO, L. - Reduccion of sudden deaths after myocardical infarction by treatment with alprenolol. Lancet, 2: 1157-1161, 1974. 